ÉGYPTE

monde arabe

\section{Égypte/Monde arabe}

$10 \mid 2013$

Les élections de la révolution (2011-2012)

\title{
Le sauvetage des monuments de Nubie par l'Unesco (1955-1968)
}

\section{Chloé Maurel}

\section{(2) OpenEdition}

1 Journals

Édition électronique

URL : https://journals.openedition.org/ema/3216

DOI : 10.4000/ema.3216

ISSN : 2090-7273

\section{Éditeur}

CEDEJ - Centre d'études et de documentation économiques juridiques et sociales

\section{Édition imprimée}

Pagination : 255-286

ISBN : 978-2-905838-81-0

ISSN : $1110-5097$

\section{Référence électronique}

Chloé Maurel, «Le sauvetage des monuments de Nubie par l'Unesco (1955-1968) », Égypte/Monde arabe [En ligne], 10 | 2013, mis en ligne le 10 février 2014, consulté le 07 juillet 2022. URL : http:// journals.openedition.org/ema/3216 ; DOI : https://doi.org/10.4000/ema.3216

Ce document a été généré automatiquement le 7 juillet 2022.

Tous droits réservés 


\title{
Le sauvetage des monuments de Nubie par l'Unesco (1955-1968)
}

\author{
Chloé Maurel
}

1 L'Unesco (organisation des Nations Unies pour l'éducation, la science et la culture), créée en 1945, a vu son action dans le domaine du patrimoine prendre de plus en plus de place dans son programme au fil du temps. Le sauvetage des monuments de Nubie, au sud de l'Égypte, à savoir les temples d'Abou Simbel, construits au temps de Ramsès II au XIII ${ }^{e}$ siècle avant notre ère, a été effectué de 1955 à $1968^{1}$. Ce sauvetage reste comme l'une des actions opérationnelles de plus grande ampleur réalisées par l'Unesco. Il lui a conféré un prestige durable.

2 La présente étude qui retrace cette campagne de sauvetage se fonde sur les archives conservées au siège de l'Unesco à Paris : rapports de l'Unesco, articles de presse, et surtout échanges de correspondance entre les protagonistes du projet.

3 L'intérêt d'une telle étude est de retracer, dans toute leur complexité, les étapes qui ont été nécessaires pour aboutir à ce sauvetage, de montrer comment l'Égypte et plusieurs autres pays, malgré les tensions politiques liées notamment à la crise de Suez et à la guerre d'Algérie, y ont collaboré, sous l'égide de l'Unesco, et comment un vaste réseau intellectuel et technique transnational s'est mis en place, associant archéologues, égyptologues, techniciens, hommes politiques, diplomates, agents culturels et en particulier agents de l'Unesco, autour de cet objectif commun. Le rôle de certains acteurs-clés, comme l'égyptologue française Christiane Desroches-Noblecourt, le ministre de la culture égyptien Saroïte Okacha, et le directeur général de l'Unesco René Maheu, sera mis en évidence. Ainsi, cette étude s'inscrit au carrefour de l'histoire des relations internationales et de l'histoire culturelle.

4 Comment l'Unesco a-t-elle été amenée à agir en faveur des temples de Nubie? Comment s'est déroulé le sauvetage de ces temples et comment les multiples difficultés ont-elles pu être surmontées? Comment s'est articulée l'action des différents protagonistes et que révèle cette campagne de sauvegarde sur la place et le rôle de l'Égypte dans les relations internationales de l'époque? Quelles ont été les répercussions de cette opération dans l'opinion publique mondiale? Il s'agira ici de 
retracer les études préliminaires, l'appel international de l'Unesco pour les monuments de Nubie, le sauvetage des temples, les difficultés multiples, le rôle des États-Unis, et le sauvetage de Philae.

\section{Les études préliminaires}

5 En 1955, Nasser, qui vient d'arriver au pouvoir en Égypte, décide de construire le haut barrage d'Assouan, d'une importance économique vitale pour ce pays dont le désert représente environ $90 \%$ de la superficie. Pour Habib Ayeb, «la construction du haut barrage d'Assouan a été un des effets positifs de la guerre froide ", et cette entreprise redessine les relations entre l'Égypte et le Soudan, indépendant depuis $1956^{2}$.

La construction du barrage d'Assouan fait aussi peser des menaces sur les temples de Nubie, puisque la mise en place du barrage doit entraîner la formation d'un immense lac artificiel de 5000 kilomètres carrés, le lac Nasser. La zone destinée à être engloutie comprend non seulement les temples d'Abou Simbel et de Philae, mais aussi d'innombrables autres monuments et témoignages historiques et artistiques datant de la préhistoire jusqu'à l'époque médiévale : gravures, inscriptions rupestres, une dizaine de temples et de chapelles rupestres, une dizaine de temples construits ${ }^{3}$.

7 Initialement, l'Unesco n'avait pas prévu de sauver les monuments de Nubie. Il s'agit au début, sur la demande du gouvernement égyptien, d'envoyer des missions d'archéologues pour faire des relevés afin de garder la trace de ces monuments avant qu'ils ne disparaissent. Sur l'impulsion de l'Unesco, des missions archéologiques de différents pays font des fouilles approfondies des différentes zones de la région. Les États participants sont très intéressés par la possibilité d'envoyer des missions, et rapidement, les demandes de concessions de fouilles affluent ${ }^{4}$. Ces fouilles donnent lieu à des résultats importants ${ }^{5}$. Cependant, de fortes rivalités se développent entre les missions nationales pour l'attribution des zones de fouilles les plus intéressantes et la délimitation de ces zones (problèmes de délimitation et de chevauchements) ${ }^{6}$. Il y a aussi parfois, sur une même zone, des rivalités entre les missions chargées de l'étude préhistorique et celles chargées de l'étude égyptologique. Ces rivalités, peu constructives, consternent Louis Christophe, agent de l'Unesco envoyé sur place : «la campagne de Nubie n'est pas une foire d'empoigne. Il y a du travail pour tous les spécialistes. [...] Ne travaillons-nous pas tous dans le même but, un but scientifique ? »?

8 La participation de l'URSS aux fouilles, annoncée mais pas confirmée, reste incertaine jusqu'en 1961, ce qui inquiète l'Unesco, qui lui a concédé le terrain de Wâdî 'Allâqî. En effet, étant donné les nombreuses offres des États d'envoyer des missions de fouille, presque toute la Nubie égyptienne est désormais répartie entre les différentes missions d'archéologues. "Il ne reste pratiquement plus de terrains à fouiller en Nubie égyptienne ; aussi ne devons-nous pas risquer que Wâdî 'Allâqî nous reste sur les bras ", comme l'observe un autre fonctionnaire de l'Unesco responsable de ce programme, M. van der Haagen ; c'est finalement très tardivement que l'URSS confirme son intention et entreprend des fouilles dans cette zone ${ }^{8}$. L'URSS devient dans ces années un acteur majeur au Moyen-Orient et notamment en Égypte : la superpuissance communiste a séduit Nasser en s'engageant à financer le barrage d'Assouan et Hélène Carrère d'Encausse parle pour les années postérieures à 1967 de «pax sovietica» au Moyen Orient ${ }^{9}$. 
9 À la supervision des fouilles s'ajoute le fait que l'Unesco contribue en 1955 à la création $\mathrm{du}$ « centre de documentation et d'études sur l'histoire de l'art et la civilisation de l'Égypte ancienne ", le CEDAE, au Caire, constitué d'archéologues, d'architectes des monuments, de dessinateurs, de photographes. L'égyptologue française Christiane Desroches-Noblecourt ${ }^{10}$, conservatrice du département égyptien au musée du Louvre, prend une part active à son élaboration, en collaboration avec le gouvernement égyptien. L'Unesco envoie des experts travailler au CEDAE et y former du personnel égyptien, et dote le centre d'un équipement moderne. Ce centre mène d'importants travaux de reconstitution documentaire sur les témoignages artistiques de la zone de Nubie menacée de submersion ${ }^{11}$. Ces travaux contribuent à la prise de conscience de l'immense richesse archéologique de cette zone, jusqu'alors mal connue à cause de sa situation excentrée, au cœur du désert. Une évolution se produit alors dans l'esprit des fonctionnaires de l'Unesco et des chercheurs participants: l'idée de sauver ces monuments émerge.

En avril 1956, sur l'impulsion de Christiane Desroches-Noblecourt, le comité international pour les monuments, les sites d'art et les fouilles archéologiques recommande à l'Unesco de lancer un appel pour le sauvetage de ces monuments. Mais la crise de Suez qui éclate quelques mois plus tard empêche l'Unesco de donner suite à cette recommandation. En 1957, au conseil exécutif de l'organisation, le représentant de l'Égypte, M. 'Awad, prône la mise en place d'une aide internationale pour la sauvegarde des monuments de Nubie, mais sans succès; la conjoncture politique y est alors défavorable, dans le contexte de la guerre d'Algérie (le Gouvernement provisoire de la république algérienne, GPRA, étant installé au Caire ${ }^{12}$, et des conséquences de l'expédition franco-britannique à Suez. À la suite de cette expédition, qui a beaucoup nui aux relations franco-égyptiennes, les fonctionnaires de l'Unesco présents en Égypte ont été assignés à résidence puis évacués en Crète par l'ONU. Les actions de l'Unesco en Égypte sont interrompues. Seule Christiane Desroches-Noblecourt poursuit ses travaux au Caire. Elle connaît cependant des dissensions importantes avec l'égyptologue égyptien Ahmad Badawî, nommé administrateur général du CEDAE, et avec le directeur de l'Institut français d'archéologie orientale (IFAO).

11 Le règlement du sort des monuments de Nubie se fait donc lentement et après beaucoup d'incertitudes et de problèmes, et grâce aux efforts de Christiane DesrochesNoblecourt, qui s'emploie activement à convaincre le gouvernement égyptien et les fonctionnaires de l'Unesco, dont plusieurs sont initialement réticents à l'idée du sauvetage de ces monuments ${ }^{13}$.

\section{L'appel international pour les monuments de Nubie}

12 En avril 1959, sur les conseils de Christiane Desroches-Noblecourt, Saroïte Okacha, le ministre de la culture égyptien, adresse au directeur général de l'Unesco, Vittorino Veronese, une demande pour que cette institution internationale devienne l'intermédiaire officiel pour obtenir une aide internationale, scientifique et technique de grande envergure afin de déplacer les monuments de Nubie susceptibles de l'être et d'intensifier les relevés documentaires pour ceux impossibles à déplacer. Le gouvernement égyptien offre de céder aux parties qui auront effectué des fouilles dans la zone menacée au moins la moitié des produits de leurs fouilles, et se déclare prêt à céder certains temples et une collection d'antiquités. Vittorino Veronese, conseillé par 
le Français René Maheu qui est favorable à une telle entreprise, accepte. Okacha, Maheu, et Christiane Desroches-Noblecourt, qui joue le rôle d'intermédiaire entre les deux, sont les trois personnalités qui, par leur motivation pour ce projet, ont joué un rôle moteur pour en permettre la réalisation malgré tous les obstacles politiques ${ }^{14}$.

L'Unesco organise alors en septembre 1959, en coopération avec le ministère égyptien de la culture, une première mission de deux spécialistes, l'Allemand Knecht et l'Italien Gazzola, ainsi qu'un voyage en Nubie pour informer archéologues, responsables de musées, historiens de l'art, architectes des monuments historiques, et experts internationaux ${ }^{15}$. L'Unesco réunit en octobre 1959 un groupe d'experts international qui dresse des listes de priorité pour les monuments à sauvegarder, en plaçant en premier Abou Simbel et Philae, affirmant que leur disparition serait «une perte irréparable pour le patrimoine culturel de l'humanité $»^{16}$. La presse internationale accorde un écho important à la demande du gouvernement égyptien, ce qui éveille l'intérêt de l'opinion publique ${ }^{17}$. En 1959 , le conseil exécutif, après des divisions sur la question de savoir s'il vaut mieux établir le principe d'une contribution financière obligatoire ou volontaire des États, choisit le principe d'une contribution volontaire (c'est-à-dire non obligatoire, son versement étant laissé à la libre initiative des États) ${ }^{18}$.

L'appel pour les monuments de Nubie est lancé par le directeur général de l'Unesco le 8 mars $1960^{19}$, au cours d'une séance présidée par André Malraux, à un moment où la conjoncture politique internationale est redevenue favorable à une telle entreprise, bien que l'Égypte n'ait pas encore renoué ses relations diplomatiques avec l'Angleterre, la Belgique et la France ${ }^{20}$. En France, l'appel est appuyé par le ministre Maurice Couve de Murville (ancien ambassadeur au Caire) ${ }^{21}$, et par André Malraux, ministre d'État chargé des affaires culturelles, qui, en mars 1960, dans un discours prononcé à l'Unesco, loue la « hardiesse magnifique » de la campagne, qui fait de la Nubie « une vallée de la Tennessee de l'archéologie $»^{22}$. Peu après, Christiane Desroches-Noblecourt contribue elle-même, par des négociations avec notamment Saroïte Okacha (alors ministre de la Culture d'Égypte), à la reprise des relations diplomatiques entre la France et l'Égypte, au moyen d'une large subvention de la France à la campagne, et de l'acceptation du gouvernement égyptien de dégeler l'argent des Français spoliés (à la suite de la nationalisation du canal de Suez en 1956 et de la crise qui a suivi) et de le mettre à la disposition de la sauvegarde ${ }^{23}$.

15 Au fil de la campagne de Nubie, le rôle joué par l'Unesco a progressivement évolué : alors que l'organisation devait initialement jouer seulement un rôle d'intermédiaire entre le gouvernement égyptien, les autres gouvernements donateurs et les entreprises qui se chargeraient de l'opération matérielle, elle s'est impliquée de plus en plus dans l'organisation du sauvetage, jouant en fait un rôle beaucoup plus important que celui d'intermédiaire. Sur la décision prise par le Comité international d'action en mai 1960, l'Unesco étend sa responsabilité à l'ensemble des travaux de sauvetage ${ }^{24}$.

\section{Le sauvetage des temples}

La campagne de Nubie a souffert de retards et de problèmes d'organisation, dus notamment à la lourdeur administrative des mécanismes mis en place (comité international d'action, comité exécutif, comités nationaux dans les différents États, comité de contrôle composé d'ingénieurs, haut comité international, comité d'honneur...), institutions créées ad hoc ${ }^{25}$. 
17 En 1962, les autorités égyptiennes craignent que «dans la masse de comités et de rapports, la campagne de Nubie ne finisse par être submergée avant les monuments eux-mêmes ${ }^{26}$. Les comités nationaux, qui sont créés par plusieurs États sur l'impulsion de l'Unesco en vue de susciter des financements, s'avèrent souvent peu efficaces, ne comportant pas assez de personnalités de la finance, de l'industrie ou de la vie économique du pays, comme le recommandait l'Unesco ${ }^{27}$. Le comité consultatif mis en place par l'Unesco n'est pas non plus efficace; il se réunit de moins en moins après 1962, à cause de l'obstruction du gouvernement égyptien, dans le cadre de la rivalité entre celui-ci et l'Unesco pour la responsabilité de la campagne ${ }^{28}$. Louis Christophe souligne le «malaise évident » qu'entraîne cette situation et estime en 1963 qu'il est « du devoir de l'Unesco d'étudier sérieusement ce problème pour suggérer au gouvernement de la RAU [République arabe unie] le moyen de sortir de l'impasse $»^{29}$. En septembre 1963, M. Gysin, agent de l'Unesco, déplore : «je dois me demander si notre comité existe toujours $\|^{30}$. Finalement, les efforts de l'Unesco aboutissent à la tenue d'une nouvelle session du Comité en décembre 1963, mais celui-ci ne joue pas le rôle de supervision qu'il aurait dû jouer ${ }^{31}$. La campagne a été rendue difficile non seulement par les problèmes de relations entre l'Unesco et le gouvernement égyptien, mais aussi par les réticences de nombreux États à participer à son financement ${ }^{32}$.

18 Malgré toutes ces difficultés administratives, la campagne avance grâce à la détermination du DG de l'Unesco, René Maheu, des agents de l'Unesco qui y œuvrent, et du gouvernement égyptien. L'appel lancé par le DG de l'Unesco en 1960, les actions de promotion comme la «semaine des antiquités de Nubie» (1963), l'exposition internationale itinérante sur Toutankhamon, et le soutien de personnalités, ont permis une prise de conscience par la communauté internationale de l'urgence de sauver les temples de Nubie et la récolte de contributions volontaires importantes ${ }^{33}$.

Le site d'Abou Simbel devient à partir de mars 1964 un gigantesque chantier international où ingénieurs, ouvriers et administrateurs de toutes nationalités travaillent de concert ${ }^{34}$. Les temples sont découpés en blocs au fil hélicoïdal (fil en forme d'hélice, inventé en 1854 par un ingénieur français, Eugène Chevalier, permettant de trancher de la pierre), soulevés un par un et déplacés. Tous les monuments, inscriptions et reliefs rupestres situés en Nubie égyptienne et soudanaise sont inventoriés, toutes les fresques de la période chrétienne sont détachées des murs et transportées en lieu sûr, tous les sites sont prospectés. Les deux grands temples sont reconstitués à 64 mètres au-dessus de l'ancien site, à l'identique et dans la même orientation, de telle sorte que le soleil puisse avoir le même jeu qu'autrefois sur la façade et à l'intérieur du sanctuaire ${ }^{35}$. En outre, de nombreux autres temples sont déplacés et reconstruits ailleurs, chacun par les soins d'un État ${ }^{36}$.

Les travaux préparatoires ont été longs et laborieux pour parvenir à la solution technique retenue. D'autres solutions, comme celle d'un barrage en terre autour des temples, d'un mur, d'un exhaussement des temples huit mètres plus haut, d'un détournement du cours du Nil par deux barrages et de l'ouverture à la dynamite d'un nouveau lit plus à l'ouest, de la mise en place d'un dock flottant, ont été envisagées mais n'ont finalement pas été retenues, soit à cause de leur coût trop élevé, soit à cause de l'aspect esthétique, soit à cause du danger. Les tenants de ces différents projets se sont longuement affrontés. Le choix du lieu où réinstaller les temples déplacés a aussi prêté à controverse : valait-il mieux les laisser en Nubie, ou les implanter plus au Nord, plus près des grands centres touristiques $?^{37}$ Christiane Desroches-Noblecourt évoque 
les «interminables discussions » et les "différends » entre spécialistes au sujet des différents systèmes envisagés, les "discussions enfiévrées" qui ont présidé à la décision du plan d'action ${ }^{38}$. En outre, la tâche était urgente, étant donné la montée des eaux du lac de retenue, ainsi que des crues exceptionnelles du Nil en août $1964^{39}$.

Il est notable d'observer que l'Unesco s'est beaucoup plus préoccupée du sort des monuments que de celui des habitants de la région; la construction du barrage entraîne en effet un déplacement massif de population, puisque l'apparition du lac Nasser oblige les Nubiens à abandonner leurs foyers et leurs terres et à être transférés vers les localités de Kom Ombo et d'Esna, où ils sont logés dans des baraquements et où leur avenir s'annonce incertain. Seul un groupe d'ethnologues égyptiens et américains mène une enquête ethnologique sur les traditions de ces populations, qui apparaissent destinées à disparaitre ${ }^{40}$; et les équipes de l'ASFEC (Arab States Fundamental Education Centre, centre éducatif de l'Unesco situé à Sirs Al-Layân en Égypte) s'efforcent de collaborer à la planification urbaine de ces nouvelles cités de Kom Ombo, mais sans grande efficacité semble-t-i ${ }^{41}$.

\section{Des difficultés multiples}

Les difficultés ont porté en particulier sur le financement de l'opération. L'Unesco s'est divisée sur les moyens d'obtenir les fonds nécessaires à l'opération : faut-il y œuvrer par une campagne de promotion dans le grand public, ou plutôt par une campagne ciblée en direction des grandes fortunes? Pour William Farr, agent de l'Unesco, il serait «entièrement irréaliste de croire que ces activités d'information et de publicité pourraient en elles-mêmes conduire a des contributions spontanées d'argent à l'échelle nécessaire ». Farr estime que la promotion en direction du grand public ne sert à rien et qu'il faut consacrer toute l'action en direction des gros donateurs potentiels ${ }^{42}$. Le prince Sadruddin Aga Khan, secrétaire exécutif du comité international d'action, est du même avis et déplore en 1962 que l'exposition Toutankhamon, si elle est « un très gros succès sur le plan culturel», " ne rapporte absolument rien à la campagne" sur le plan financier alors qu'elle avait été conçue en partie pour rapporter des fonds ${ }^{43}$. Quant à Constantin Christofidis, professeur d'histoire de l'art à l'université de Syracuse aux États-Unis, chargé par son université d'une mission à Abou Simbel, il incite l'Unesco à renoncer à "viser à atteindre l'Américain moyen, parce que l'Américain moyen ne s'intéresse pas assez aux choses de l'art », mais à concentrer la "propagande » sur les milieux universitaires américains ${ }^{44}$.

Finalement, l'Unesco mène son action de promotion dans différentes directions. Le "Comité américain pour la campagne de Nubie » promeut la campagne de façon très organisée, en direction des différents publics, en utilisant les journaux, les revues, la radio, la télévision, mais aussi les institutions culturelles, l'industrie, les canaux éducatifs, les groupes civiques. Cependant, des obstacles persistent, comme l'observe M. Lassalle, directeur du Comité américain, soulignant «l'apathie des égyptologues américains pour le projet, parfois transformée en ouverte hostilité ", la mauvaise image relative de l'Égypte aux États-Unis, et «l'opinion prévalente qu'une aide étrangère, intensifiée de façon énorme dans les derniers mois par la création d'agences gigantesques à Washington, serait une obligation "morale"des citoyens américains pour élever le standard de vie des populations dans les pays les moins privilégiés "; en outre, les activités de l'Unesco seraient perçues comme "désorganisées et confuses » 
par l'opinion américaine ${ }^{45}$. Un des facteurs qui ont diminué la réussite de la campagne de promotion est l'exagération systématique à laquelle se livre l'Unesco dans ses publications et brochures de promotion, notamment au sujet de la valeur des objets découverts ${ }^{46}$.

Au début de l'année 1963, l'Unesco lance un nouvel appel aux États membres pour combler le déficit du budget destiné à la Nubie. Plusieurs États y répondent, poussés principalement par la volonté d'en retirer du prestige ; c'est le cas de la France, dont le but est de "tire[r] un regain de prestige qui pourrait faciliter [son] action culturelle", et de ne pas se laisser éclipser par les autres pays comme les États-Unis, la RFA, les PayBas, le Royaume-Uni ${ }^{47}$. Les États rivalisent ainsi dans les fouilles et dans l'aide financière ${ }^{48}$. Ce sont les États-Unis qui sont les plus actifs dans ces deux domaines ${ }^{49}$.

Comme l'évoque Michel Prévost, ancien fonctionnaire de l'Unesco, tout au long de la campagne de Nubie se poursuit "une campagne de dénigrement» de la part de nombreux journalistes; l'Unesco s'emploie inlassablement à les "rassurer ", à les convaincre que l'opération est bien en cours et va réellement aboutir; elle y œuvre notamment par l'organisation de voyages de journalistes sur le Nil ${ }^{50}$. L'Unesco s'efforce de désamorcer les réticences souvent très importantes et tenaces de l'opinion et des États, par de nombreux voyages de hauts fonctionnaires de l'Unesco, qui vont directement parlementer, avec conviction et détermination, avec les fonctionnaires gouvernementaux et avec les personnalités concernées, dans les États ${ }^{51}$.

Par ailleurs, cette campagne de Nubie a donné lieu à toutes sortes de difficultés au cours de son déroulement : erreurs, malentendus, désaccords, critiques, rivalités entre États... Ainsi, l'Unesco a financé entre 1960 et 1962 d'importantes fouilles en vue de retrouver le tombeau d'Alexandre le Grand, à partir de documents qui sont des faux fournis par un imposteur, Stelio Comoutsos. Cette imposture contribue à discréditer l'Unesco aux yeux des archéologues, comme l'observe l'égyptologue français Louis Christophe ${ }^{52}$. Cette campagne donne lieu aussi à des détournements d'argent ${ }^{53}$. Par ailleurs, le nettoyage des temples d'Abou Simbel, effectué avec trop peu de soin, entraîne l'effacement de nombreuses traces intéressantes, et fait perdre de leur éclat aux couleurs ${ }^{54}$; en outre, des dommages sont faits sur certains blocs, entraînant l'apparition de fissures ${ }^{55}$.

Une des ambiguïtés de cette opération a été le fait que l'Égypte semblait «vendre » ses temples antiques aux États, se débarrasser de son patrimoine culturel pour mener à bien son développement économique. Pour riposter à ce reproche lancé par Israël, l'Unesco et le gouvernement égyptien ont bien pris soin d'éviter le terme de "vente ". En échange de leur participation au sauvetage, les États reçoivent des temples et des objets en "contrepartie ", il ne s'agit pas d'une "transaction commerciale", d'une "dissociation du patrimoine culturel égyptien » mais bien plutôt d'une "extension nouvelle de son rayonnement $\|^{56}$. Dès avril 1959, Christiane Desroches-Noblecourt déplore dans une lettre à Maheu "des pressions de toute sorte» intervenues concernant les dons d'objets aux États ${ }^{57}$. Ceux-ci suscitent rapidement l'intérêt de nombreuses institutions, notamment américaines (musées et universités), qui se manifestent alors auprès de l'Unesco pour proposer leur participation ${ }^{58}$.

28 Ainsi, en échange de leur participation au sauvetage et au déplacement de ces temples, les États-Unis se voient offrir les temples de Derr et de Dendur, la France celui d'Amada (ce qui se fait avec beaucoup de difficultés techniques et de retards) ${ }^{59}$, la RFA celui de Kalabchâ (cette opération se fait avec beaucoup de succès) ${ }^{60}$. La perspective de recevoir 
des temples Nubiens suscite en certains cas l'enthousiasme de la population, comme c'est le cas de la ville d'Indio, en Californie, très désireuse de recevoir le temple de Derr; une association "Temple Derr for Indio" est créée, menant campagne pour l'obtention de ce temple, faisant valoir que par son climat d'oasis dans le désert, et par sa topographie, qui se rapprochent de ceux de la Nubie, la ville d'Indio conviendrait particulièrement bien à une telle transplantation. L'Unesco n'est pas opposée à un tel transfert, mais le seul problème est de «trouve[r] le moyen d'accepter cette offre sans qu'elle fasse figure d'achat ${ }^{61}$. Beaucoup de complications et de retards se produisent ${ }^{62}$. De même, en 1965, McCullough, directeur du comité national américain pour la campagne, explique à Ali Vrioni, fonctionnaire de l'Unesco et directeur de la campagne de Nubie, que les musées américains sont très intéressés par l'idée d'aider la campagne de Nubie par des dons, et même par de fortes sommes, mais seulement à condition qu'ils reçoivent, en échange de leurs dons, des objets d'antiquités de valeur ; c'est donc d'un achat déguisé qu'il s'agit ${ }^{63}$. Son successeur à la tête de ce comité, M. Lassalle, s'inquiète lui aussi de la mise en place d'" une sorte d'opération de chantage » de la part des institutions américaines pour l'obtention des contreparties ${ }^{64}$.

De la part du gouvernement égyptien, motivations économiques et motivations patrimoniales entrent en concurrence; en effet, pour des raisons économiques, les terrains soumis aux fouilles doivent être rapidement mis en culture, dans le cadre du programme national de récupération agricole. Pour permettre cela, un instrument moderne est testé qui permet de sonder la terre à la recherche de vestiges, sans la creuser; cette méthode présente l'avantage d'être rapide et de permettre de mettre une terre en culture rapidement si elle est jugée ne pas contenir de vestiges archéologiques ${ }^{65}$.

Le déroulement des fouilles connaît des difficultés à cause de rivalités entre États, par exemple entre l'Égypte et la RFA ${ }^{66}$, et entre l'Égypte et les États-Unis ${ }^{67}$. La campagne donne lieu aussi à une collaboration parfois harmonieuse entre États, comme entre le Soudan et l'Égypte ${ }^{68}$. Pourtant, cela n'était pas gagné d'avance étant donné qu'une longue inimitié opposait l'Égypte et le Soudan, la Nubie apparaissant comme une pomme de discorde entre eux (« la Nubie antique était l'ennemie intime de l'Égypte», écrit Marc Goutalier) ${ }^{69}$.

Des malentendus et des désaccords, de nature juridique, se produisent entre l'Unesco et le gouvernement égyptien au sujet de la signature des contrats, en 1961: le gouvernement égyptien exige que l'Unesco devienne partie contractante, et fournisse une contribution prélevée sur son propre budget ; or cela a été exclu par l'Unesco ${ }^{70}$. Les relations difficiles entre l'Unesco et le gouvernement égyptien s'expliquent en partie par la diversité des interlocuteurs de l'Unesco au sein du gouvernement égyptien, interlocuteurs qui n'ont pas tous la même position sur le sujet, et en particulier par une dissension entre Saroïte Okacha et Chihâta Âdam ${ }^{71}$, et par la démission en 1962 du prince Sadruddin Khan, qui était un des fervents soutiens de la campagne ${ }^{72}$. En outre, de vives oppositions à l'encontre du projet se développent au sein de la commission nationale égyptienne pour l'Unesco; son président, Kamâl Husayn, qui est aussi ministre de l'Éducation nationale, est hostile à la campagne ${ }^{73}$.

Il semble néanmoins que la campagne ait, par la coopération internationale qu'elle a impliquée, contribué à un apaisement des tensions entre États arabes et États occidentaux $^{74}$. Ainsi, il apparaît que cette campagne patrimoniale a permis d'aplanir les 
relations, restées jusque là teintées d'hostilité réciproque, entre d'un côté l'Égypte et d'un autre côté la France et le Royaume-Uni à la suite de l'expédition de Suez de 1956.

\section{Le rôle important des États-Unis}

L'évolution progressive de la position des États-Unis vis-à-vis de la campagne de Nubie a contribué au succès de celle-ci. Les États-Unis étaient initialement hostiles à l'idée de participer à son financement ${ }^{75}$, de même que l'URSS, qui estimait que puisqu'elle a assumé le financement du haut barrage d'Assouan, elle n'aurait pas à contribuer au sauvetage des monuments ${ }^{76}$. L'Unesco mène une tâche longue et persévérante de persuasion auprès du gouvernement américain et des riches milieux privés américains, en particulier au moyen du très dynamique "comité américain pour la préservation d'Abou Simbel " (American Committe to Preserve Abu Simbel). L'Unesco et ce comité ne négligent aucun effort pour séduire ces milieux : diffusion de rapports et de coûteuses photographies couleurs à l'intention des milieux spécialisés, conférences, rencontres, projections de films, expositions à l'intention du grand public, exposé des intérêts économiques de l'opération à l'intention des milieux économiques, création de vêtements de luxe et de bijoux sur le thème de l'Égypte pour toucher les milieux fortunés ${ }^{77}$, etc.

Ces efforts s'avèrent fructueux ${ }^{78}$. L'exposition "Les Trésors de Toutankhamon ", inaugurée en novembre 1961 à Washington, est un grand succès. Elle reçoit une couverture exceptionnelle de la part de la presse et de la télévision, elle donne lieu à de nombreux lunchs officiels rassemblant d'importantes personnalités comme Jacqueline Kennedy. Partout où elle circule aux États-Unis, elle est apparemment un succès «colossal» et contribue à une prise de conscience importante de la part de la population américaine ${ }^{79}$. La diffusion du film Cléopâtre, qui sort en 1962, est assortie, aux États-Unis, d'un court-métrage sur les monuments de Nubie avant le générique, ainsi que d'un message d'Elisabeth Taylor au public du monde entier en faveur de la campagne $^{80}$. Cette campagne se révèle un succès dans le public américain ${ }^{81}$. De nombreuses entreprises américaines se montrent très intéressées ${ }^{82}$. L'action de collecte de dons est très fructueuse dans ce pays, mais son transfert se heurte à des problèmes juridiques (taxes, devises), ce qui cause des retards ${ }^{83}$.

35 Le gouvernement américain est de plus en plus intéressé par la campagne au fil du temps. En février 1961 se déroule au département d'État une conversation confidentielle entre Vittorino Veronese et le secrétaire d'État américain, concernant les aspects politiques de la campagne. Veronese s'efforce de convaincre les États-Unis de participer, faisant valoir les avantages politiques et financiers qu'ils en retireraient ${ }^{84}$. Le Congrès américain, initialement hostile à la campagne, tend à s'y rallier à partir du message de Kennedy au Congrès à ce sujet en $1961^{85}$. Cette année-là cependant, les relations politiques entre les États-Unis et l'Égypte sont très tendues, à cause de la question d'Israël et de la politique de la RAU de non-alignement ${ }^{86}$.

En novembre 1963 sont signés deux accords, l'un entre l'Unesco et le gouvernement égyptien, l'autre entre l'Unesco et les États contractants. Mais des doutes subsistent alors toujours sur la contribution des États-Unis, et sur le sort des temples d'Abou Simbel, puisque le gouvernement américain refuse de signer l'accord, subordonnant cette signature à l'accord préalable du Congrès ${ }^{87}$. En mars 1963, Paulo de Berrêdo Carneiro, président du comité exécutif, et René Maheu adressent un télégramme 
solennel et pathétique au président des États-Unis, lui demandant instamment de soutenir la campagne, soulignant l'urgence, étant donné la montée des eaux ${ }^{88}$. En juin 1963, le président Kennedy approuve la participation des États-Unis au projet de sauvetage des temples. Il y a à cela un intérêt économique pour cet État, puisque sa participation sera versée en livres égyptiennes obtenues de la vente du blé américain à la RAU ${ }^{89}$. Ce n'est qu'en octobre 1964 que le représentant américain au conseil exécutif annonce enfin officiellement la décision positive du gouvernement américain ${ }^{90}$.

En 1965, Harry C. McPherson, assistant secrétaire d'État américain, se dit convaincu de l'intérêt pour les États-Unis de participer au sauvetage des temples. En effet, une telle aide " augmenterait les occasions pour les Américains d'influencer les idées et actions dans le monde arabe en direction de la modération, de la coopération et de la stabilité ${ }^{91}$. De même, en 1967, le président Johnson se félicite de la réussite du comité américain pour la campagne de Nubie à récolter d'importantes sommes et affirme que cette campagne constitue "un excellent exemple de partenariat créatif entre le gouvernement égyptien et l'industrie américaine $»^{92}$.

\section{Des découvertes fructueuses}

Les fouilles donnent lieu à de nombreuses découvertes : ainsi, la mission archéologique polonaise découvre une basilique copte, en pierre et briques rouges, aux murs recouverts de fresques byzantines $d u \mathrm{VII}^{\mathrm{e}}$ siècle, enfouie à 20 mètres sous les vestiges d'une forteresse islamique du XVII ${ }^{e}$ siècle, près de la ville de Faras au Soudan ${ }^{93}$. La mission de Turin découvre treize tombes rupestres dans la région de Dehmit, des graffitis rupestres préhistoriques, et des inscriptions hiéroglyphiques sur des roches ${ }^{94}$. La mission de l'université de Chicago met au jour une nécropole de la protohistoire, au sud d'Abou Simbel, jamais ouverte encore; les missions de Boston et de Yale découvrent la tombe d'un chef Nubien, Heka-Nefer, dans la région d'Ikhmindi; la mission italienne met au jour une ville nobade (Nubie chrétienne), édifiée entièrement en pierres sèches ${ }^{95}$; l'Américain Simpson découvre, sur le site de Toschké-Ermenneh, deux statuettes funéraires dans la tombe d'un prince d'Aniba du Nouvel Empire nommé Heka-Nefer, en schiste, très bien conservées ${ }^{96}$.

Toutefois, les rivalités se déchaînent concernant la paternité des découverte archéologiques; ainsi en 1963, Louis Christophe déplore que "les savants américains publient leurs rapports dans des revues étrangères "; « cela est tout-à-fait contraire à l'esprit dans lequel nous travaillons ». Il estime qu'il faudrait plutôt « grouper dans une publication unique toutes les recherches effectuées en Nubie ». " Malgré mes efforts, je n'arrive pas, vous le constaterez, à obtenir de mes collègues qu'ils respectent [...] les termes de leur contrat $»^{97}$.

\section{L'inauguration des temples d'Abou Simbel}

Les temples d'Abou Simbel, entièrement reconstruits, sont inaugurés en septembre 1968, lors d'une cérémonie au pied des temples dans leur nouveau site. Si les déplacements des petits temples (et notamment celui de Kalabchâ), ont connu souvent des retards ${ }^{98}$, en revanche le sauvetage d'Abou Simbel est terminé en avance sur les prévisions (vingt mois plus tôt) ${ }^{99}$. À leur inauguration, René Maheu, directeur général de l'Unesco, prononce un discours emphatique ${ }^{100}:$ « Nous sommes venus, Ô Roi, ajouter 
notre travail au tien pour préserver ta quête d'éternité. [...] Nous avons pieusement dressé ta gigantesque majesté et recomposé la suave beauté de ta reine avec l'escorte hiératique des divinités tutélaires. [...] Grâce aux efforts de tous, te voici, sauf, prêt à reprendre, intact, sur la barque d'Ammon, ton voyage au long des siècles vers le soleil levant de chaque lendemain ${ }^{101}$. En outre, à l'inauguration, il est donné lecture d'un chapitre du $\operatorname{Coran}^{102}$. Ainsi, l'Unesco confère à cette inauguration une connotation quasi-religieuse.

\section{Le sauvetage de l'île de Philae}

41 La sauvegarde de Philae peut être évoquée plus brièvement car elle a été menée sur le modèle de celle d'Abou Simbel. L'île de Philae, surnommée "la perle de l'Égypte ", comporte un temple d'Isis, une colonnade et un édifice de l'époque de Trajan. Cet ensemble, situé en aval du haut barrage d'Assouan, et en amont de l'ancien barrage, est submergé pendant les deux-tiers de l'année. Les variations incessantes du niveau de l'eau exercent sur la pierre une action d'usure très grave. En 1908, Pierre Loti avait écrit un roman sur la détérioration progressive de ces temples, La mort de Philae.

Dès 1955 est envisagée l'idée d'isoler l'île pour la sauver ${ }^{103}$. Sous l'impulsion de l'Unesco, plusieurs projets en ce sens sont étudiés à partir de 1959. Celui qui est initialement retenu consiste à réunir les îlots par une série de petits barrages mettant ainsi à l'abri de la montée des eaux l'île de Philae, de préférence à un autre projet prônant le démontage des temples puis leur remontage sur la même île après l'avoir exhaussée de quelques mètres. Finalement c'est une autre solution qui est préférée : le transfert des monuments sur l'île voisine d'Agilka, plus élevée ${ }^{104}$.

En 1968, René Maheu lance un appel pour le sauvetage de Philae, sur le modèle de celui lancé pour Abou Simbel. Des comités spécialisés sont créés, et des contrats sont établis, de manière analogue à ce qui avait été fait pour la campagne précédente. Le financement est obtenu beaucoup plus facilement que pour Abou Simbel, bénéficiant du précédent réussi que constituait cette opération. En revanche, la mise en œuvre concrète de l'opération entraîne des "discussions sans fin et des incidents de toute sorte » comme le souligne Christiane Desroches-Noblecourt ${ }^{105}$.

Ce n'est qu'en 1972 que commencent les travaux. La guerre du Kippour en 1973 entraîne leur interruption provisoire. Ils connaissent des difficultés (pénurie de matériel, dysfonctionnements dans l'organisation du travail) et prennent un important retard ${ }^{106}$. Un problème qui a contribué à affecter cette opération a été le remplacement en cours de route d'une grande partie de ses responsables : ainsi, René Maheu quitte l'Unesco en 1974, Nasser meurt en septembre 1970 ; Okacha est écarté du pouvoir par le successeur de Nasser, Anouar Al Sadate; M. van der Haagen, G. Rosi, Piero Gazzola, fonctionnaires de l'Unesco qui ont participé activement aux deux campagnes, meurent avant la fin de celle de Philae ${ }^{107}$. Malgré tout, le sauvetage des temples de Philae est mené à bon port et achevé en 1978.

La campagne de Nubie, menée et réussie malgré de nombreuses difficultés logistiques, administratives, et politiques, a constitué un grand succès pour l'Unesco. Ce succès a contribué à rehausser son image de marque. En 1968, au moment de l'inauguration des temples d'Abou Simbel, l'Unesco est au summum de sa popularité et connaît son âge d'or, sous la direction de René Maheu. Le sauvetage des temples de Nubie a infléchi 
l'orientation postérieure des actions de l'Unesco vers la protection du patrimoine. En 1972, l'organisation adopte la convention internationale sur le patrimoine naturel et culturel mondial, et à partir de cette date est constituée la liste des sites classés au patrimoine mondial de l'Unesco, liste qui est devenue aujourd'hui une des activités les plus médiatiques et les plus réussies de l'institution.

De plus, cette campagne de Nubie révèle un aspect remarquable de la politique égyptienne sous Nasser, à savoir la préoccupation de ce pays pour son patrimoine. Cette préoccupation entre parfois en conflit avec l'objectif de développement économique, mais les autorités égyptiennes s'efforcent d'articuler les deux. En outre, par cette action patrimoniale qui prend des dimensions internationales, l'Égypte assied sa place sur la scène internationale : cette campagne permet à l'Égypte d'entretenir et même d'améliorer ses relations avec certains pays comme la France, et d'accroître sa visibilité et son rayonnement dans le monde, notamment grâce à l'intense campagne de promotion menée parallèlement au sauvetage des temples.

\section{BIBLIOGRAPHIE}

Aboukorah O. \& Leturcq J.-G. (dir.), 2009, « Pratiques du patrimoine en Égypte et au Soudan », Égypte/Monde arabe, vol. 3, n 5-6.

AYEB H., 2001, « L'Égypte et le barrage d'Assouan. Que serait l'Égypte sans ce très grand barrage? ", Hérodote, vol. 4, n 103, p. 137-151.

BENHAMOU F., 2012, Économie du patrimoine culturel, Paris, La Découverte.

BOURREAU G., 2002, La politique française à l'Unesco durant la période gaullienne, 1958-69: l'Unesco, un instrument pour le prestige français, maîtrise d'histoire, Paris I Panthéon-Sorbonne.

CARRÈRE D'ENCAUSSE H., 1975, La politique soviétique au Moyen-Orient, 1955-1975, Paris, Presses de Sciences-Po.

DESROCHES-NOBLECOURT Ch., 1992, La Grande Nubiade, ou le parcours d'une égyptologue, Paris, StockPernoud.

DU Bourguet P., 1960, « Pourquoi sauver les monuments de Nubie ?», Études, 1960, p. 247-255.

DUPONT-MONOD C., 2002, «Christiane Desroches-Noblecourt, la pharaonne », L'Histoire, n² 271, p. 28.

FOGEL F., 1995, « Des Nubies, des Nubiens : traditions scientifiques et locales de l'identité », Égypte/ Monde arabe, vol. 1, $\mathrm{n}^{\circ} 24$, p. 75-86.

- 1997, Mémoires du Nil. Les Nubiens d'Égypte en migration, Paris, Karthala.

GOUTALIER M., 2010, « La proximité et ses limites dans les relations égypto-soudanaises », Confluences Méditerranée, vol. 4, n 75, p. 197-211.

MAUREL Ch., 2005, L'Unesco de 1945 à 1974, thèse de doctorat d'histoire contemporaine, Paris I

Panthéon-Sorbonne.

- 2010, Histoire de l'Unesco. Les trente premières années (1945-1974), Paris, L'Harmattan. 
NAFAA H., 1977, L'Égypte et l'Unesco, thèse de science politique, Paris I Panthéon-Sorbonne.

PREVOST M., 1996, L'île des Uneskimos, Mémoires d'un ancien fonctionnaire de l'Unesco, 1949-1983, dactylographié, non publié, conservé aux archives de l'Unesco.

TRINTIGNANT H., 1974, La protection internationale des biens culturels en temps de paix, thèse de droit public, Montpellier.

\section{NOTES}

1. Sur la protection du patrimoine en Égypte, cf. Omnia Aboukorah et Jean-Gabriel Leturcq (dir.), «Pratiques du patrimoine en Égypte et au Soudan », Égypte/Monde arabe, vol. 3, n 5-6, 2009, et en particulier l'article d'Aymé Lebon, "Réutilisation des monuments historiques à des fins didactiques, culturelles ou artistiques». Sur le patrimoine culturel en général, cf. Françoise Benhamou, Économie du patrimoine culturel, Paris, La Découverte, 2012, qui analyse le patrimoine culturel comme un bien culturel global.

2. Habib Ayeb, «L'Égypte et le barrage d'Assouan. Que serait l'Égypte sans ce très grand barrage?", Hérodote, vol. 4, n 103, 2001.

3. En 1842 est construit le barrage de Muhammad 'Alî au nord du Caire. C'est le premier d'une série dont le plus important a été le premier barrage d'Assouan, construit par des ingénieurs anglais en 1902. Des besoins croissants en eau et en terres nouvelles ont amené les autorités égyptiennes à élever le niveau de ce barrage à deux reprises, en 1912 et en 1934.

4. Archives de l'Unesco, 069 (62) N/A 02 (100), II : lettre de van der Haagen à Christophe, 7 avril 1961 ; 069 (62) /A 02 (100) CA12/86, III : lettre de Christophe à Gysin, $1^{\text {er }}$ fév. 1963 ; exposé du représentant du directeur général de l'Unesco devant le comité consultatif, 2-5 déc. 1963. Dans cet article, sauf mention contraire, les documents d'archives mentionnés viennent des archives de l'Unesco.

5. 069 (62) N/Christophe : note de Christophe, $n^{\circ}$ 1664, 25 sept. 1962 : Christophe estime que « la campagne internationale de sauvegarde des monuments nubiens peut d'ores et déjà être, sur le plan spécialement archéologique, considérée comme un succès »; 14 pays ont envoyé une ou plusieurs missions scientifiques pour faire des fouilles en Nubie, et plusieurs ont fourni des experts au centre de documentation. Il observe que "toute la Basse-Nubie est partagée entre les différentes missions de fouilles», et qu'" une grande partie du travail est déjà achevée ».

6. En 1961 les institutions savantes se renvoient la balle pour la réalisation de l'étude préhistorique de la Nubie égyptienne, qui ne les intéresse pas (069 (62) Nubie : 93 A 52 (62) : lettre de Christophe à Jan van der Haagen, 29 mars 1961).

7. 069 (62) N/Abou Simbel/A 02. CA 120/29, II : lettre de Christophe à Zbynek Zaba, 18 déc. 1963.

8. 069 (62) Nubie, IV : déclaration de M. Piotrowski, 28 mai 1960 ; lettre de Jan van der Haagen à Ali Vrioni, 28 avril 1961 ; lettre de Maheu à A.G. Koulagenkov, 13 juin 1961.

9. Hélène Carrère d'Encausse, La politique soviétique au Moyen-Orient, 1955-1975, Paris, Presses de Sciences-Po, 1975 ; chapitre III : « Pax Sovietica ». 
10. Sur Christiane Desroches-Noblecourt, cf. Clara Dupont-Monod, «Christiane Desroches-Noblecourt, la pharaonne ", L'Histoire, $\mathrm{n}^{\circ}$ 271, 2002, p. 28.

11. L'équipement consiste en laboratoires photographiques, en une agence d'architecture antique et en des bureaux d'enregistrement et de recherches ; l'Unesco se charge aussi de l'équipement technique du bateau-laboratoire. Hassan Nafaa, L'Égypte et l'Unesco, thèse de science politique, Paris I Panthéon-Sorbonne, 1977, p. 564-567.

12. Ibid., p. 567.

13. Christiane Desroches-Noblecourt, La Grande Nubiade, ou le parcours d'une égyptologue, Paris, Stock-Pernoud, 1992, p. 130-133, 139-140, 143-148, 152-164, 169-179 : elle convainc Maheu, Jan van der Haagen, l'Égyptien Hannâ Sabâ, alors conseiller juridique de l'Unesco, Mustafa 'Âmir, administrateur général du CEDAE, et Louis Christophe ; 069 (62) N/Christophe : note de Christophe, $n^{\circ} 1664,25$ sept. 1962 ; 062 (62) Nubie I : note confidentielle de J. Thomas du 29 mai 1959 ; compte rendu de la réunion du 17 juin 1959.

14. Michel Prévost, L'île des Uneskimos, Mémoires d'un ancien fonctionnaire de l'Unesco, 1949-1983, 1996, dactylographié, non publié, conservé aux archives de l'Unesco, p. 74-75. Cf. 54 EX/24, p. 2 ; Ch. Desroches-Noblecourt, op. cit., p. 180-181. "Ce grand patriote, lettré, passionné de musique classique, soucieux d'enrichir constamment ses déjà vastes connaissances, était un militaire de carrière, fils d'un général et compagnon de Nasser, dont il fut le "pilier culturel"». Okacha avait aussi été un officier de la Révolution égyptienne ; 062 (62) Nubie, I : mémorandum de Jan van der Haagen au DG, 30 juill. 1959 ; X 07.83 Veronese, II : Le Journal d'Égypte, 2 fév. 1960, p. 1-2 : «Pour sauver les trésors de la Nubie »; "Le président a reçu hier M. Veronese ». La presse égyptienne fait l'éloge du ministre de la culture égyptien, S. Okacha, de sa « lutte de diplomatie, de ténacité et d'enthousiasme inlassable au service de cette Idée», de son "don de persuasion », de « conviction ».

15. H. Nafaa, op. cit., p. 568-570. Cf. doc 54/EX/24, annexe ; et doc 55/EX 7, annexe 11 ; 062 (62) Nubie, I : lettre de Saroïte Okacha à Veronese, 6 avril 1959; Ch. DesrochesNoblecourt, op. cit., p. 180-199.

16. $54 \mathrm{EX} / 24$, p. 2 ; $54 \mathrm{EX} /$ décision $4-7$; $55 \mathrm{EX} / 7$, annexe 1, p. 6-7, cité dans H. Nafaa, op. cit., p. 571-572.

17. H. Nafaa, op. cit., p. 573, 575 : 55 EX/SR 19, p. 208.

062(62) Nubie, I : réponse de Veronese à Saroïte Okacha, non datée.

18. 61 EX/SR.25, p. 305 ; cité dans H. Nafaa, op. cit., p. 584 ; 60 EX/SR 11, p. 92, cité dans H. Nafaa, op. cit., p. 578-579.

19. Cf. un article publié peu après cet appel : Pierre du Bourguet, « Pourquoi sauver les monuments de Nubie? ", Études, 1960, p. 247-255.

20. Ch. Desroches-Noblecourt, op. cit., p. 213 ; H. Nafaa, op. cit., p576-577.

21. Archives diplomatiques françaises, série Cabinet du ministre, Couve de Murville, carton 180 : lettre de M. Couve de Murville à Veronese, 8 mars 1960 ; note pour le Cabinet du ministre de la DGACT, 21 nov. 1962, ; docs. cités par Gaëlle Bourreau, La politique française à l'Unesco durant la période gaullienne, 1958-69: l'Unesco, un instrument pour le prestige français, maîtrise d'histoire, Paris I Panthéon-Sorbonne, 2002, p. 33-35. 
22. Archives Unesco, dossier biographique d'André Malraux; discours d'A. Malraux, "L'acte par lequel l'homme arrache quelque chose à la mort », 8 mars 1960 (reproduit dans le Courrier de l'Unesco, mai 1960, p. 9-11).

23. Ch. Desroches-Noblecourt, op. cit., p. 216.

24. 069 (62) N/Abou Simbel/A 02, I : lettre de Maheu à Okacha, 27 avril 1962; H. Nafaa, op. cit., p. 576, 602. Cf. 11 C/PRG/9, p. $6 ; 069(62) \mathrm{N} /$ Christophe : lettre de Veronese à Okacha, 18 juill. 1960: en juill. 1960, Veronese écrit à Okachaque le Comité international d'action, en mai 1960, a estimé que l'Unesco ne devrait pas se limiter au rôle d'intermédiaire mais que la responsabilité de l'Unesco devrait s'étendre à toute l'entreprise jusqu'à l'achèvement des travaux ; Veronese y est favorable.

25. Le comité consultatif d'experts est chargé d'étudier les offres de participation transmises au gouvernement égyptien, de le conseiller sur leur emploi et sur la répartition des contreparties offertes en reconnaissance de l'aide internationale $(\mathrm{H}$. Nafaa, op. cit., p. 601) ; H. Nafaa, op. cit., p. 607-609; 069 (62) N/Abou Simbel/A 02, I : rapport du comité de contrôle du projet de surélévation des temples d'Abou Simbel, $1^{\mathrm{e}}$ session, Paris 9-13 oct. 1961; Al-Ahrâm, 11 oct. 1961 : «Cinq ingénieurs internationaux seront chargés d'établir les conditions nécessaires à la surélévation d'Abou Simbel »; Al-Ahrâm, 8 déc. 1961: "Un haut comité international présidé par Hasan Zakî pour superviser les opérations de sauvegarde d'Abou Simbel»; 069 (62) /A 02 (100) CA12/85A, I : 22 déc. 1959: rapport de réunion dans le bureau du DG: le comité international sera composé de 20 ou 30 personnalités choisies en fonction de l'équilibre géographique et culturel, et appartenant au monde de la culture, de la technique et de la finance ; de nombreuses personnalités importantes (rois, reines...) sont contactées pour faire partie du comité international (nombreuses lettres de Veronese); mémo de Maheu au DG, 24 fév. 1960 ; US National Commission, Newsletter, 23 janv. 1961, vol. VIII, n², p. 1 ; H. Nafaa, op. cit., p. 599.

26. 069 (62) N/Abou Simbel/A 02, I : lettre de Vrioni à J.K. van der Haagen, 12 avril 1962. 27. (62) /A 02 (-4) CA12/98 : lettre d'Ali Skalli au DG, 14 avril 1960 ; liste des membres du Comité national suisse, et récapitulatif des comités créés au 21 nov. 1960 ; lettre de Vrioni à Thomas, 23 juin 1960.

28. 069 (62) /A 02 (100) CA12/86, III : lettre du service des monuments de la Nubie à Louis Christophe, 18 mars 1963 ; lettre de Gysin à Christophe, 19 juill. 1963 ; lettre de Christophe au service des monuments de Nubie, 11 août 1963.

29. 069 (62) /A 02 (100) CA12/86, III : lettre de Christophe au service des monuments de Nubie, 13 août 1963: "Tout se passe comme si le gouvernement de la RAU voulait mettre le Comité consultatif devant un fait accompli : la signature du contrat pour le découpage d'Abou Simbel afin de ne plus remettre en question le problème de sa sauvegarde "; lettre de Vrioni à Christophe, 14 août 1963.

30. 069 (62) /A 02 (100) CA12/86, III : lettre de Gysin à Christophe, 5 sept. 1963.

31. 069 (62) /A 02 (100) CA12/86, III : lettre de Christophe au Service des monuments de Nubie, 31 août 1963 ; lettre de Christophe au Service des monuments de Nubie, 18 nov. 1963 ; 069 (62) N/A 02 (100), II : lettre de Veronese à Hermann J. Abs, 6 avril 1961.

32. En 1959, la correspondance entre J. van der Haagen et Ch. Desroches Noblecourt témoigne des difficultés de communication et des tensions entre l'Unesco et le gouvernement égyptien (ex : 069 (62) Nubie A 06 : lettre de van der Haagen à Desroches Noblecourt, 26 oct. 1959). 
33. 069 (62) N/Christophe : Al-Ahrâm, 31 août 1963 : «Eugene Black présidera aux USA la campagne de donations pour la sauvegarde des monuments de Nubie»; 062 (62) Nubie, I : réunion d'experts internationaux pour la sauvegarde des sites et monuments de la Nubie menacée, Le Caire, 1-11 oct. 1959 ; 069 (62) N/Christophe : Al-Ahrâm, 3 avril 1963 : « Un appel adressé aux membres du Rotary dans le monde entier par le ministre de la Culture et de l'Orientation nationale en faveur de la sauvegarde des temples d'Abou Simbel. Le ministre invite un demi-million de Rotariens à participer à la sauvegarde »; 069 (62) N/Christophe : lettre de Christophe au service des monuments de la Nubie, 13 avril 1963 ; Al-Ahrâm, 10 avril 1963 : « Le Congrès des Antiquités à Tunis étudiera "La semaine des Antiquités de Nubie" "; Le Progrès égyptien, $1^{\mathrm{er}}$ mai 1963 : "L'Irak apportera une large contribution au sauvetage des monuments de Nubie. Une "semaine" sera consacrée à ce projet dans tous les pays arabes"; 069 (62) N/Abou Simbel/1 114. CA 120/97: note de M. Wallett, 20 janv. 1965; les époux Kennedy soutiennent la campagne.

34. H. Nafaa, op. cit., p. 611.

35. Hélène Trintignant, La protection internationale des biens culturels en temps de paix, thèse de droit public, Montpellier, 1974, p. 103-107 ; 069 (62) N/Christophe : Le Progrès égyptien, 23 fév. 1963: "Les marbriers de Carrare attendus bientôt au Caire pour soumettre aux responsables leur projet de sauvetage d'Abou Simbel ».

36. 069 (62) N/Christophe : Al-Ahrâm, 24 avril 1963 ; Le Progrès égyptien, 18 avril 1964 : « Un musée archéologique serait créé à Assouan ».

37. 062 (62) Nubie, I : lettre de Georges Post à Okacha, 12 sept. 1959; IV : réunion d'experts internationaux pour la sauvegarde des sites et monuments de la Nubie menacée, Le Caire, 12 nov. 1959, compte rendu; 069 (62) N/Christophe: Le Progrès égyptien, 17 fév. 1963 : « Sous le signe d'Abou Simbel »; Al-Ahrâm, 24 avril 1963 ; 069 (62) N/Abou Simbel/A 02, II : Le Progrès égyptien, 6 juin 1963 : « Hier, sous la présidence de M. Hasan Zakî, première réunion des experts chargés de l'examen des projets de sauvetage des temples d'Abou Simbel »; 069 (62) /A 02/17 CA12/85A : compte rendu de la réunion d'experts internationaux concernant la sauvegarde des sites et monuments de la Nubie ancienne, 20 sept. 1959 ; 069 (62) Nubie/Abou Simbel, II : documents techniques, comme le rapport du comité de spécialistes scandinaves du 2 juin 1961 ; télégramme de Saroïte Okacha à Maheu, 20 juin 1961 ; note confidentielle du 21 juin 1961 ; Le Progrès égyptien, 17 juin 1961; Le Monde, 22-23 janv. 1961 : « À l'Académie des inscriptions et des Belles Lettres: la sauvegarde du temple d'Abou Simbel»; Le Monde, 24 janv. 1961: «À l'Académie des inscriptions et des Belles Lettres: M. Schaeffer dénonce le danger du projet italien pour la sauvegarde d'Abou Simbel »; New Scientist, n² 234, 11 mai 1961 : lettre de l'égyptologue A.J. Arkell ; H. Nafaa, op. cit., p. 582-586 ; H. Trintignant, op. cit., p. 92-94.

38. Ch. Desroches-Noblecourt, op. cit., p. 200-210, 270-272.

39. 069 (62) N/Christophe : lettre de Christophe au service des monuments de la Nubie, 13 avril 1963 ; Ch. Desroches-Noblecourt, op. cit., p. 270-271.

40. Nubie/BUR, 1967-86: Nubie/13, annexe I, p. 12-13 : déclaration du représentant de la RAU, lors de la $13^{\mathrm{e}}$ session du comité exécutif, 14-16 mars 1967; 069 (62) N/ Christophe : Al-Ahrâm, 5 nov. 1961 : «L'émigration des habitants de Nubie commencera en juillet 1963 »; Ch. Desroches-Noblecourt, op. cit., p. 236 : elle évoque ces populations « vouées à une réinstallation dans de tristes cités à Kom Ombo et autour d'Assouan ». 
41. Archives de l'ONU, RAG-2/330, box 108 : lettre de Bogdan Theodorovic à PerezGuerrero, non datée, 4 p., p. 3-4: «poor planning ». Sur les Nubiens, cf. Frédérique Fogel, Mémoires du Nil. Les Nubiens d'Égypte en migration, Paris, Karthala, 1997 ; F. Fogel, "Des Nubies, des Nubiens: traditions scientifiques et locales de l'identité », Égypte/Monde arabe, vol. 1, n²4, 1995.

42. 069 (62) /A 02 (-4) CA12/98 : lettre de William Farr à Vrioni, 14 déc. 1961.

43. 069 (62) /A 02 (-4) CA12/98 : mémo confidentiel de Vrioni à van der Haagen, 23 mars 1962.

44. 069 (62) N/Christophe : Le Progrès égyptien, 15 fév. 1963 : « Une porte où frapper : les universités américaines pourraient encore financer le sauvetage d'Abou Simbel ».

45. 069 (62) N/A 02 (73) : mémorandum de Lassalle, 26 nov. 1961 : «Draft for a public relations program ».

46. 069 (62) N/A 02 (73) : mémorandum de Lassalle à Salsamendi, 13 déc. 1961 ; lettre de Lassalle à van der Haagen, 8 janv. 1962.

47. $12 \mathrm{C} / 6,4.1$; archives diplomatiques françaises, série cabinet du ministre, Couve de Murville, 180 : note pour le cabinet du ministre de la DGACT, Francois Charles-Roux, 18 janv. 1964 ; note de la DGACT sur la sauvegarde du temple d'Amada, 24 mars 1964 (docs cités par G. Bourreau, op. cit., p. 33-35).

48. 56 EX/SR 8, p. 62-63 ; cité dans H. Nafaa, op. cit., p. 580 ; H. Nafaa, op. cit., p. 587 ; 069 (62) Nubie/Abou Simbel, II : lettre confidentielle de Maheu à Saroïte Okacha, 2 août 1962.

49. 56 EX/SR 8, p. 62-63 ; cité dans H. Nafaa, op. cit., p. 580 ; 069 (62) Nubie Abou Simbel, III : lettre d'A. Vrioni au secrétaire de la délégation permanente de l'Espagne, 23 janv. 1963 ; H. Nafaa, op. cit., p. 617.

50. M. Prévost, L'île des Uneskimos, op. cit., p. 76-78.

51. H. Nafaa, op. cit., p. 613-614; 069 (62) Nubie Abou Simbel, III : mémo de J. Thomas à Maheu, 15 juill. 1959 : en 1959, l'Unesco ayant appris que James Rorimer, directeur du Metropolitan Museum of Art de New York avait « certains doutes et appréhensions à l'égard de l'intervention de l'Unesco ", envoie Jean Thomas pour le faire changer d'avis, le convaincre. Les deux hommes ont " un entretien très long et très franc ", au cours duquel Thomas estime avoir réussi à "rassurer » Rorimer ; 069 (62) N/Christophe : AlAhrâm, 6 janv. 1963: "Les délégués de l'Unesco visitent les pays pour les inviter à la coopération en faveur de la sauvegarde des monuments »; 069 (62) Nubie Abou Simbel, III : Le Monde, 27 janv. 1962, p. 9: «Pour des raisons techniques et financières, la décision du gouvernement égyptien de mettre en adjudication les travaux d'Abou Simbel semble prématurée »; 069 (62) N/A 02 (73) : Lassalle à van der Haagen, 8 janv. 1962 : Lassalle souligne l'hostilité générale des milieux des musées et des égyptologues aux États-Unis pour la campagne de Nubie et est exaspéré de voir que l'Unesco ne veut pas l'admettre.

52. 069 (62) N/Christophe : lettre de Christophe à Asabuki, 16 janv. 1962; lettre de Christophe à Rosi, 15 mars 1962.

53. 069 (62) N/Christophe : mémo de Christophe à van der Haagen, 12 mars 1962.

54. 069 (62) N/Christophe : lettre de Christophe à Vrioni, 5 déc. 1962.

55. 069 (62) N/Abou Simbel/A 02. CA 120/29, III : comité de contrôle, $13^{\mathrm{e}}$ session, 16 nov. 1966 ; mais globalement l'opération de découpage et de transport est satisfaisante 
(069 (62) N/Abou Simbel/A 02. CA 120/29, III : lettre de Christophe à V. Nenadovic, 25 oct. 1967 ; lettre de Christophe à Chihâta Âdam, 19 janv. 1968).

56. 069 (62) /A 02/17 CA12/85A: rapport confidentiel de la réunion d'experts internationaux concernant la sauvegarde des sites et monuments de la Nubie ancienne, non daté ; rapport de la réunion d'experts internationaux concernant la sauvegarde des sites et monuments de la Nubie ancienne, 20 sept. 1959.

57. 062 (62) Nubie, I : lettre de Ch. Desroches-Noblecourt à Maheu, 8 avril 1959.

58. 069 (62) Nubie, IV : plusieurs institutions américaines (ex.: Peabody Museum, University of California, The Bruce Museum dans le Connecticut) écrivent à l'Unesco en nov. 1959 ; 069 (62) N/Christophe : lettre de Vrioni à Christophe, 17 janv. 1961 ; 069 (62) N/A 114/113 (73): lettre de Froelich Rainey, directeur du musée de l'université de Pennsylvania, à Maheu, 15 mars 1960 ; lettre de Froelich Rainey à Saroïte Okacha, 14 oct. 1960.

59. Ch. Desroches-Noblecourt, op. cit., p. 238-245. La France prend en charge le temple d'Amada, monument d'aspect mixte (moitié grotte, moitié construction par assises de pierre), grâce aux démarches de Desroches-Noblecourt auprès du général de Gaulle ; les études pour le déplacement de ce temple prennent du retard à cause des incidents franco-égyptiens de déc. 1961 ; les experts français ont du mal à se décider entre divers procédés (déplacement par translation, par flottaison, ou encore démontage et remontage sur un nouveau site). Finalement la France commence le déplacement dans la seconde moitié de l'année 1964 (069 (62) Nubie, IV: CUA 10803, 29 juill. 1963; déclaration de $\mathrm{M}$. Cain à la $60^{\mathrm{e}}$ session du conseil exécutif ; lettre de relance du ministre de la culture égyptien, Abdel Kader Hatem, 5 août 1963 ; lettre de van der Haagen à Maheu, 6 mars 1964 ; lettre d'Olivier de Sayve à Vrioni, 22 juin 1964).

60. 069 (62) N/Christophe: Le Progrès égyptien, 28 avril 1963: «Le gouvernement américain étudie le projet de la RAU tendant au sauvetage d'Abou Simbel. Une décision rapide doit être prise à ce sujet, déclare le sous-secrétaire du département d'état américain "; Al-Ahrâm, 2 déc. 1963 ; 069 (62) N/Abou Simbel/1 114. CA 120/97 : message du Président Johnson sur Abou Simbel, 29 avril 1967; X07.21/43-15 A562 : Germany, Briefing for DG, non signé ni daté ; Ch. Desroches-Noblecourt, op. cit., p. 236-237.

61. 069 (62) N/A 875 (73) : lettre de Yassu Gauclère à Christophe, 22 fév. 1961.

62. 069 (62) N/A 875 (73) : lettre de la Indio Chamber of Commerce au DG, 16 déc. 1960 ; lettre de Yassu Gauclère à Christophe, 22 fév. 1961 (citation) ; note manuscrite anonyme; réponse de Wilson à la Indio Chamber of Commerce, 17 janv. 1963. La correspondance se poursuit pendant plusieurs années entre l'Unesco et l'association "Temple Derr for Indio »; lettre de 'Abd Al-Mun'im Al-Sâwî, sous secrétaire d'État de la RAU, à Maheu, 19 avril 1964 ; lettre de Chihâta Âdam au ministre de la Culture et de l'Orientation nationale de RAU, 9 juin 1964.

63. 069 (62) N/Abou Simbel/1 114. CA 120/97 : lettre de Mc Cullough à Vrioni, 8 juin 1965 ; réponse de Vrioni à Mc Cullough, 8 juill. 1965.

64. 069 (62) N/A 02 (73) : mémo confid. de Lassalle à Salsamendi, 2 avril 1962.

65. 069 (62) N/Christophe : Le Progrès égyptien, 2 mai 1963 : «Une nouvelle méthode de recherche archéologique va bientôt être appliquée en Égypte et dans le Moyen Orient : le "Mighty Midget"(moustique puissant) épargnera effort, temps et argent ».

66. En déc. 1961, la presse égyptienne affirme que 50 des pierres du temple de Kalabchâ ont été détruites pendant leur débarquement en Nubie à cause de l'inexpérience des 
ouvriers chargés du débarquement de ces pierres (069 (62) N/Philae, III : Al-Ahrâm, 15 déc. 1961: "Les pierres du temple de Kalabchâ ont subi un accident grave. La destruction de 50 pierres antiques pendant leur débarquement en Nubie»). Le gouvernement de RFA dément et dénonce les "mauvaises intentions» de la presse égyptienne (lettre de Christophe à van der Haagen, 20 déc. 1961). J. van der Haagen estime que cette campagne calomnieuse dans la presse est due à l'hostilité de la presse égyptienne contre M. Choukry et contre S. Okacha (lettre de van der Haagen à G. Bersu, 26 déc. 1961). En fév. 1962, Okacha fait insérer dans la presse un rectificatif et un communiqué élogieux sur les efforts du gouvernement de RFA dans sa participation au projet (lettre de Okacha au DG, $1^{\text {er }}$ fév. 1962).

67. 069 (62) N/A 114/113 (73) : lettre de Desroches-Noblecourt, 3 sept. 1962. Des lettres de Christophe confirment ce jugement.

68. 069 (62) N/Philae, III : lettre de Christophe au service des monuments de Nubie, 5 déc. 1962.

69. Marc Goutalier, "La proximité et ses limites dans les relations égyptosoudanaises ", Confluences Méditerranée, vol. 4, n 75, 2010.

70. 069 (62) N/Abou Simbel/A 02, I : réponse de Okacha, 3 oct. 1961; lettre de Maheu à Okacha, 22 sept. 1961 ; lettre d'Okacha au DG, 3 août 1961 ; lettre de Okacha au DG, 16 sept. 1961.

71. 069 (62) N/Abou Simbel/A 02, I : lettre de Vrioni à J. van der Haagen, 12 avril 1962 : Vrioni estime avoir la « certitude » que les lettres adressées par l'Unesco à Okacha sont en fait « étudiées uniquement par Shehata Adam ».

72. 069 (62) N/Abou Simbel/A 02, I : lettre de Vrioni à J. van der Haagen, 12 avril 1962; 069 (62) /A 02 (-4) CA12/98: mémo confidentiel du 23 mars 1962, de Vrioni à van der Haagen ; archives diplomatiques américaines, Decimal file, RG59, entry CDF 1960-63 : box 822 : mémorandum de conversation avec Sadruddin Khan et John Barrow, 15 fév. 1961.

73. 069 (62) Nubie Abou Simbel, III : mémo de la division des musées et monuments à Maheu, du 22 sept. 1959, et du 23 sept. 1959.

74. 069 (62) N/A 57 Nubie : $4^{e}$ conférence archéologique de la Ligue arabe, à Tunis, 17-29 mai 1963, rapport de L. Gomes Machado, 20 juin 1963.

75. M. Prévost, op. cit., p. 80.

76. Archives privées de Vittorino Veronese, institut Luigi Sturzo, carton 32 : lettre de J. Thomas à Veronese, 4 sept. 1960.

77. 069 (62) N/A 02 (73) : New York Times, 20 janv. 1962 : «Land of Nile New Source For Fashion »; lettre de la Commission nationale américaine à Lassalle, 23 janv. 1962.

78. 069 (62) N/Abou Simbel/A 02. CA 120/29, II : lettre de Vrioni à Maheu, 24 mai 1962: Vrioni fait un voyage en Espagne où il s'efforce, par des conversations avec diverses personnes, d'influencer le gouvernement espagnol pour qu'il donne un crédit supplémentaire pour Abou Simbel; Veronese, carton 32: lettre de J. Thomas à Veronese, 4 sept. 1960 : J. Thomas affirme avoir «trouvé beaucoup de changement depuis un an " dans la position du département d'état américain et du congrès américain au sujet de la Nubie ; 069 (62) N/A 380.383/858, II : mémo de Christophe au service des monuments de Nubie, 29 fév. 1964 ; lettre de Christophe à l'administrateur général du centre de documentation, 19 nov. 1963 ; 069 (62) N/Abou Simbel/1 114 : lettre d'Ali Vrioni à Elmandjra, 4 fév. 1965 ; rapport «The rescue of the Temples, a 
statement prepared for The Old Dominion Foundation, by The American Committe to Preserve Abu Simbel », 12 avril 1965; ex. : efforts auprès de Westinghouse Electric International, Radio Corporation of America, The Bank of America, The Ford Motor Company, The Getty Oil Company, The Singer Company, Sacony-Mobile Oil Company ; le comité américain comprend des grands noms des milieux industriels, savants, politiques, du spectacle, intellectuels, universitaires, etc.; correspondance entre Max Mc Cullough, directeur du comité américain, et Ali Vrioni, directeur de la Campagne de Nubie (1965); lettre de Rodolphe J.A. de Seife à Vrioni, 4 août 1966 ; lettre de Rodolphe J.A. de Seife à Vrioni, 7 oct. 1966 ; lettre de Seife à Vrioni, 14 déc. 1966 ; lettre de Seife à Maheu, 10 fév. 1967 ; lettre de Seife à Maheu, 23 fév. 1967 ; Maheu à Seife, 14 mars 1967 ; 069 (62) N/A 02 (73) : lettre de F. Reinhardt à Okacha, 9 août 1960; Okacha à F. Reinhardt, 16 août 1960 ; compte rendu du meeting du US national committee for the preservation of the Nubian monuments, 14 oct. 1961 ; lettre de Lassalle à Muhammad Habîb, 18 déc. 1961.

79. 069 (62) N/A 02 (73) : lettre de John A. Pope à Lassalle, 7 nov. 1961; lettre de Brew à Maheu, 5 fév. 1962 ; lettre de Lassalle à van der Haagen, 8 déc. 1961.

80. 069 (62) /A 02 (-4) CA12/98 : mémo confidentiel de Vrioni à van der Haagen, 23 mars 1962.

81. 069 (62) /A 02 (-4) CA12/98 : lettre de J.O. Brew à Veronese, 9 sept. 1960 ; archives diplomatiques américaines, Decimal file, RG59, entry CDF 1960-63 : box 824 : lettre de G. Shuster à Dean Rusk, 15 déc. 1961, p. 4-5; ibid., box 827 : lettre confid. de G.N. Shuster à Dean Rusk, $1^{\text {er }}$ oct. 1962.

82. 069 (62) N/A 114/113 (73) : note de Christophe, 4 mars 1961.

83. 069 (62) N/Abou Simbel/1 114 : lettre de Rodolphe J.A. de Seife à Vrioni, 4 août 1966 ; lettre de Seife à Vrioni, 28 fév. $1967 ; 069$ (62) N/A 114/113 (73) : note de Louis Christophe, 28 nov. 1960.

84. Archives diplomatiques américaines, Decimal file, RG59, entry CDF 1960-63 : box 822 : mémorandum de conversation, confid., entre le secrétaire d'État et Veronese, 16 fév. 1961.

85. 069 (62) N/A 114/113 (73) : lettre de Christophe à van der Haagen, 9 sept. 1961 ; lettre de Okacha à Maheu, 16 oct. 1961 ; Le Progrès égyptien, 21 oct. 1961 : « M. Humbert Humphrey, membre de la commission des affaires étrangères du Sénat américain, actuellement au Caire, discutera avec les autorités responsables des projets de sauvetage des monuments de Nubie »; Le Progrès égyptien, 22 oct. 1961 : «Entretiens de M. Humphrey avec le Dr. Okacha et M. H.Z. Sabrî̀; Le Progrès égyptien, 20 mai 1964 : "Le refus de la commission budgétaire américaine de participer au sauvetage des temples égyptiens. Une attitude hostile à la RAU, mais qui nuira au patrimoine archéologique du monde entier »; Al-Akhbâr ; lettre de Veronese à Philip H. Coombs, 17 avril 1961 ; Congrès, 26 sept. 1961, nº 171 ; lettre confid. d'Edmundo Lassalle à Maheu, 28 sept. 1961 ; New York Times, 13 sept. 1961 : «House Unit Kills Funds For Egypt's Monuments ».

069 (62) N/A 02 (73) : New York Times, 28 sept. 1961 : « US Votes Funds to Save Nile Arts » ; lettre de John A. Wilson à Maheu, 29 sept. 1961 ; lettre de Lassalle à van der Haagen, 2 fév. 1962 ; mémo confid. de Lassalle à Salsamendi, 2 avril 1962 ; Veronese, carton 32 : discours de Veronese, «L'Unesco face aux problèmes d'aujourd'hui », 8 mai $1961 ; 56$ EX/ SR 8, p. 62-63, cité dans H. Nafaa, op. cit., p. 580. 
86. Archives diplomatiques américaines, box 824 : télégramme de Badeau (Le Caire) au secrétaire d'État, 20 nov. 1961.

87. H. Nafaa, op. cit., p. 589-592.

88. 069 (62) Nubie Abou Simbel, III : télégramme de Carneiro et Maheu au président des États-Unis, 8 mars 1963 : « anxiously ».

89. 069 (62) Nubie Abou Simbel, III : Al-Ahrâm, 18 juin 1963.

90. H. Nafaa, op. cit., p. 593.

91. 069 (62) N/Abou Simbel/1 114 : lettre de Harry C. McPherson à Hartford, non datée : " the American interest in the preservation of the temples of Abou Sembel »; « it increases opportunities through which Americans can influence ideas and actions in the Arab world in the direction of moderation, cooperation and stability ".

92. 069 (62) N/Abou Simbel/1 114 : message du Président Johnson sur Abou Simbel, 29 avril 1967: " an outstanding example of creative partnership between your Government and American industry ».

93. 069 (62) Nubie, IV : Unesco Features, 15 mars 1963; Unesco Features, 11 fév. 1964 ; lettre de la commission nationale polonaise pour l'Unesco à Maheu, 23 mars 1972.

94. 069 (62) Nubie, IV : dossier Turin CA 120/37.

95. 069 (62) N/A 114/113 (73) : Le Progrès égyptien, 13 avril 1963 : « La tombe découverte en Nubie par la mission américaine date de 1500 av. J.C. »; Ch. Desroches-Noblecourt, op. cit., p. 233.

96. 069 (62) N/A 114/113 (73) : rapport de mission de Christophe à Toschké-Ermenneh, 12 mars 1961.

97. 069 (62) N/A 114/113 (73) : mémo de Louis Christophe au service des monuments de Nubie, 16 fév. 1963 ; l'Américain Solecki a publié son rapport de ses recherches en Nubie dans la revue Man, vol. 62, nov. 1962.

98. 069 (62) N/Philae, III : mémo $\mathrm{n}^{\circ} 795$; télégramme $\mathrm{n}^{\circ}$ 1227, 29 janv. 1965. L'inauguration du temple de Kalabchâ, prévue initialement en mai 1964, est repoussée à fév. 1965.

99. Ch. Desroches-Noblecourt, op. cit., p. 272.

100. H. Nafaa, op. cit., p. 595-596.

101. DG/68/14, p. 2-3.

102. Ch. Desroches-Noblecourt, op. cit., p. 330-331.

103. H. Trintignant, op. cit., p. 95-96 ; Courrier de l'Unesco, oct. 1961, p. 16-20 ; numéro spécial sur Philae en décembre 1964 ; déc. 1968, p. 48-55 ; fév. 1960, p. 34-37.

104. 069 (62) N/Philae, III : lettre de Christophe au service des monuments de Nubie, 23 oct. 1966 ; Ch. Desroches-Noblecourt, op. cit., p. 211-212 ; 069 (62) /A 02/17 CA12/85A : rapport confidentiel de la réunion d'experts internationaux concernant la sauvegarde des sites et monuments de la Nubie ancienne, 12 nov. 1959 ; en 1960 le gouvernement des Pays-Bas fait des études préliminaires pour le sauvetage de Philae. La mise en service du nouveau barrage d'Al-Sadd-Al-'Âlî, entraînant des oscillations journalières rapides de 6 mètres au niveau des temples de Philae, aura pour conséquence si rien n'est fait de scinder les temples en deux au niveau de l'eau. Les Pays-Bas proposent de construire trois digues et une station de pompage. (069 (62) N/Philae, II : "Exposé sommaire des études préliminaires réalisées sur l'initiative du gouvernement des Pays- 
Bas en vue de la protection de l'île de Philae », oct. 1960). En 1964, il est décidé que les Pays-Bas assureront les travaux de sauvegarde du temple de Philae, à partir de 1967, par la construction de deux barrages qui permettront le retrait de l'eau et l'assèchement complet du temple (069 (62) N/Philae, II : Le Progrès égyptien, 2 nov. 1964 : «Les Pays-Bas assumeront les travaux de sauvegarde du temple de Philae. L'opération commencera en 1967 et coûtera six millions de livres ») ; H. Nafaa, op. cit., p. 597-611, 618-619.

105. Ch. Desroches-Noblecourt, op. cit., p. 397-428; H. Nafaa, op. cit., p. 597 ; Nubie/BUR, 1967-86: Nubie/17, p. 6, annexe 2 : appel de Maheu pour la sauvegarde des temples de Philae, 6 nov. 1968.

106. H. Nafaa, op. cit., p. 597 ; Nubie/BUR, 1967-86: UNESCO/ NUBIA/CE/XXII/3 (rapport sur la sauvegarde des monuments de Philae présenté par le gouvernement d'Égypte : état d'avancement des travaux exécutés depuis mars 1973, 9 nov. 1973, p. 3 : " observations relatives aux retards »).

107. Ch. Desroches-Noblecourt, op. cit., p. 397-406.

\section{RÉSUMÉS}

L'Unesco, créée en 1945, a vu son action dans le domaine du patrimoine prendre de plus en plus d'importance dans son programme au fil du temps. Cette organisation a supervisé le sauvetage des monuments de Nubie (temples d'Abou Simbel notamment) au sud de l'Égypte de 1955 à 1968. Ce sauvetage s'est fait grâce à la coopération de nombreux pays. Il s'inscrit dans le cadre de la politique internationale menée par Nasser et révèle l'attention de celui-ci pour le patrimoine égyptien. Il a accru le prestige de l'Égypte, ainsi que de l'Unesco, sur la scène internationale.

Unesco, created in 1945, developed along the years a growing action in the field of world heritage. It supervised the saving of the Nubian monuments (Abu Simbel notably) in the South of Egypt from 1955 to 1968. It was done thanks to the cooperation of many countries. This action is part of the international policy led by Nasser and it reveals Nasser's interest for heritage. It increased the prestige of Egypt and of Unesco on the international stage.

\section{INDEX}

Mots-clés : Égypte, Unesco, patrimoine, coopération internationale, Abou Simbel

Keywords : Egypt, Unesco, Heritage, International cooperation, Abu Simbel

\section{AUTEUR}

\section{CHLOÉ MAUREL}

Chloé Maurel, normalienne, agrégée d'histoire, docteur en histoire contemporaine de l'université Paris I, chercheuse associée à l'IHMC (CNRS/ENS), est l'auteur notamment de : Histoire de l'Unesco. 
Les trente premières années (1945-1974), Paris, L’Harmattan, 2010. Ses champs de recherche sont l'histoire culturelle, l'histoire des Nations unies et l'histoire globale.

Chloé Maurel has a PhD in contemporary history (Paris I Panthéon-Sorbonne). She is an associate researcher at IHMC (CNRS/ENS). She published a book on the history of Unesco: Histoire de l'Unesco. Les trente premières années (1945-1974), Paris, L'Harmattan, 2010. She works in cultural history, history of the UN, and global history. 